\title{
Surface Reconstruction from Point Clouds
}

\author{
Bill Toll (btoll@css.tayloru.edu) \\ Computing and System Sciences, Taylor University, Upland, IN \\ Fuhua (Frank)Cheng (cheng@cs.engr.uky.edu) \\ Computer Science, University of Kentucky, Lexington, KY
}

Key words: surface reconstruction, point clouds, feature points, reverse engineering

Abstract: Surface reconstruction from point clouds is an important element of reverse engineering as used to create solid models of existing objects. The research presented in this paper assumes no knowledge of relationships among the elements of the point cloud. The approach to initial mesh creation is based on extraction of points, called "feature points", from the cloud based on geometric relationships with nearby points. A neighborhood is established for each data point, the points in the neighborhood are ordered, and feature points are identified based on relationships among the neighbors. Feature points within a neighborhood are linked to construct a polygonal mesh that is then triangulated.

\section{INTRODUCTION}

Solid models of surfaces are desired for many purposes including input of existing physical parts to $\mathrm{CAD} / \mathrm{CAM}$ systems for modification, automatic creation of machine tool paths, error inspection, and input to finite element systems. If such models do not exist, they may be created from point clouds sampled from the surface by technologies such as laser scanners.

The creation of a model from a point cloud typically begins with the reconstruction of the surface in the form of a piecewise linear mesh surface. Although some situations allow the use of known properties of the data, the abstract problem was first stated by Hoppe, et $\mathrm{al}^{1}$. Their formulation views the problem as the process of converting an input set of $3 \mathrm{D}$ points, sampled from or near a surface, into a polygonal mesh.

One general approach to the problem creates a dense initial mesh that is simplified by one of several techniques including application of an energy function that measures deviation from the original (constructed) $\mathrm{mesh}^{2}$. Another technique ${ }^{3}$ uses bounded approximation to construct a surface mesh with each vertex within a prescribed distance from a vertex in the original mesh. The new mesh is constructed by merging faces of the original mesh. Another technique ${ }^{4}$ merges faces by triangle decimation (iterative removal of vertices).

Other approaches begin with an initial mesh and extract a surface representation directly from the mesh. The work of Hoppe's group ${ }^{1}$, for example, creates a mesh of all data points and extracts a surface from the mesh by contour tracing a signed distance function to approximate tangent planes. The vertices of the resulting triangulation are not necessarily part of the original data set, but may be created points. This triangulation, however, requires simplification as presented in following work by the same group where the mesh is optimized ${ }^{2}$ and a piecewise smooth (tangent plane continuous) surface is constructed by subdivision from the optimized triangular mesh ${ }^{5}$.

An alternate approach ${ }^{6}$ is to construct a three-dimensional Delaunay triangulation of volume elements, smooth the surface triangulation, and compute smooth Bernstien-Bezier patches to approximate the triangulated surface. A very recent approach ${ }^{7}$ constructs an output mesh that interpolates, rather than approximates, the input points by use of $3 \mathrm{D}$ Voronoi diagrams. This technique functions best when it is possible to control the sampling density over the surface.

The original version of this chapter was revised: The copyright line was incorrect. This has been corrected. The Erratum to this chapter is available at DOI: 10.1007/978-0-387-35392-0_40 


\section{FEATURE POINT BASED RECONSTRUCTION}

The focus of this work is to prepare an initial mesh that is smaller than a dense initial mesh, but is an accurate representation of the surface and at least comparable to reduced meshes produced by other techniques. The mesh that is created can be a representation of the surface or the starting point for a mesh optimization or surface fitting algorithm. One of the characteristics that differentiates the feature point approach is that it is based on the identification of points in the original data set that represent the surface topology and shape. Therefore, all the points in the resulting mesh are from the original data set and the surface, at this point in the process, approximates the surface as a well-chosen subset.

Conceptually, the approach attempts to identify "feature curves" that are natural edges of the surface. These curves are constructed by connecting feature points. The feature points are selected by a point-driven algorithm without previous mesh construction. The algorithm consists of pre-processing, feature point extraction, and mesh generation.

\section{ALGORITHM}

The first step is pre-processing where the data is input, organized, and partially analyzed to prepare for the remaining operations. Pre-processing includes data input, sorting, determination of a neighborhood for each point, and computation of an approximate normal vector to the surface at each point. The second step is extracting feature points from the data. This process is accomplished by detecting areas where there is an obvious edge based on the distribution of points in the neighborhood or areas where there is significant variation in approximate surface normals within the neighborhood. The final step of mesh generation consists of extraction of additional points as needed, generation of edges, generation of polygons from the edges, and triangulation of the polygons.

\subsection{Neighborhoods}

Since extraction of feature points from a data set is based on examination of the local variation of the surface in the vicinity of the point, a neighborhood is established for each point. A neighborhood of a point consists of data points from the original data set which are "near" the given point.

An ideal definition of neighborhood for the purpose of feature point extraction would be that a neighborhood includes only those data points that describe the surface at the given point but includes all points necessary for measurement of the variation of the surface around the given point.

Due to the above considerations and the potentially varying density of data points, a neighborhood is chosen for each point that includes all points within a given distance of the point in combination with a limit on the maximum number of points in a neighborhood.

\subsection{Normal Vectors}

Normal vectors are defined in a statistical sense for each data point based on the points in its neighborhood. The method used to find the normal vector is similar to that used by other surface reconstruction algorithms that make use of the tangent plane as an approximation to the surface in the neighborhood. The normal is calculated by applying principle component analysis on the covariance matrix of the neighbor points around the point being investigated. The normal vector is used to establish a tangent plane, but this plane is used only for sorting neighboring points rather than surface approximation.

Some surface reconstruction techniques ${ }^{1}$ replace the data point with the centroid of the neighborhood and have the additional requirement that the surface normals for all 
neighborhoods must be consistently oriented in that they all point "out". This problem has been shown to be NP-complete ${ }^{8}$. The feature point technique uses the normal and tangent plane only for sorting and, thus, does not require a globally consistent orientation.

\subsection{Feature Point Extraction}

Feature points are extracted from the data set based on variation among the points in a neighborhood. Therefore, neighboring points must be compared with each other. There are two types of comparisons used, both of which compare "adjacent" points. Therefore, the concept of adjacent points in a 3D neighborhood must be defined. The approach used is to project the points to the tangent plane for the neighborhood where the tangent plane is defined to be perpendicular to the normal vector. The projected points are then sorted by angle in the plane so adjacency relationships can be determined. Figure 1 illustrates this process.

There are three basic types of feature points and three corresponding extraction mechanisms.

Boundary feature points are data points that are selected because analysis of the points in the neighborhood indicate that there is a boundary, or natural edge, of the surface near the point.

Variation feature points are selected by a mechanism that indicates that the surface changes shape near the point. The extraction is based on the amount of variation of approximate surface normals around the data point.

Finally, there may be regions of a surface where no "interesting" shape transitions occur. The surface is smooth and uniform, but not flat. For such surfaces, it is common that no boundary or variation feature points would be extracted. Some points need to be selected from this surface so that the region is not represented as a flat region bounded by boundary and variation feature points. Such points are labeled complementary feature points.

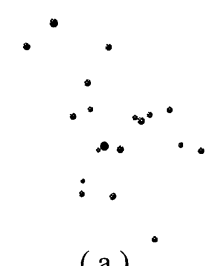

( a )

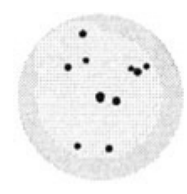

(b)

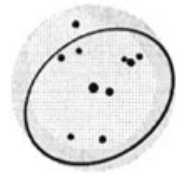

(c)

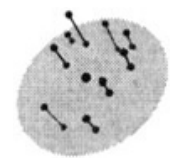

(d)

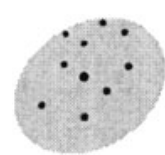

( e )

Figure 1. Data points and projection (a) data points, (b) neighborhood, (c) projection plane,

(d) points projected to plane, (e) projected points

\subsection{Boundary Feature Points}

Boundary points are feature points that are identified, or extracted, from the original data set, based on the property that points in its neighborhood indicate that an edge of the actual surface is present in the neighborhood. Furthermore, the identified point is, itself, a part of this edge. 
Such points are identified based on the spatial distribution of projected neighbor points. It is assumed that if a natural edge exists that passes through the data point, then there will be points in the neighborhood that lie along the natural edge. If there is a line formed in the projection plane, that line is assumed to indicate the presence of an edge of the surface. Line in this context means two adjacent neighbor points which when connected through the prospective feature point form an approximately straight line. Thus, if there are adjacent points in the neighborhood which have an angle between them greater than a given value, typically $160^{\circ}$, then the point is labeled a feature point. Figure 2 illustrates a boundary feature point determination.

\subsection{Variation Feature Points}

Variation feature points are extracted based on variation in surface normals within a neighborhood around a point. However, the normals calculated for each point are not used. Rather, a series of triangles are formed by using the central point as one vertex of each triangle and using two adjacent neighbor points as the other vertices for a given triangle. The surface normal for each triangle is calculated with the cross product of the vectors from the central point to the two adjacent neighbors. Note that the $3 \mathrm{D}$ neighbor points are used as the projected points all lie in the same plane. Figure 3 illustrates this process by displaying the normal vectors for the triangles in a neighborhood.

If there is sufficient variation between normals for adjacent triangles, the central point is labeled a variation feature point. While this technique will identify some points that would have been previously extracted as boundary feature points, boundary points from a flat or nearly flat surface would not be detected with the variation approach.
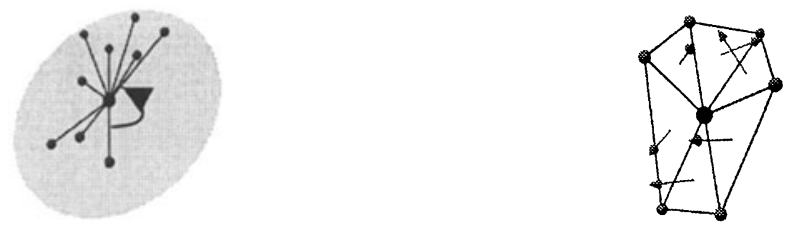

Figure 2. Testing for boundary feature point Figure 3. Testing for variation feature point

\subsection{Complementary Feature Points}

Regions of a surface where no boundary or variation feature points exist may be flat and could be represented by large triangles in a mesh. However, since there is no prior knowledge of the surface, it is not known if the surface is flat or is smoothly curved such that no variation feature points are detected. An example of such a surface is the side of a cylinder.

A point is labeled a complementary feature point if it is not a feature point and there are no feature points in its neighborhood.

It is also important that all feature points have at least two feature points in their neighborhood so that the mesh generated by connecting feature points forms closed polygons. If a feature point does not have at least two feature point neighbors, additional points must be identified. These added points can also be viewed as complementary feature points.

\subsection{Mesh Generation}

The mesh is created from edges formed by connecting feature points. A feature point should be connected to all feature points in its neighborhood. It is not necessary that all feature points in a common neighborhood are connected to each other. Indeed, if all such 
edges are formed, there will be intersecting edges. Before the edge data structure is built, intersecting edges are disallowed by removing feature points from neighborhoods that cause intersecting edges. Additional complementary points may need to be labeled so that closed polygons are formed.

Edges are generated by connecting each feature point to all feature points remaining in its neighborhood. The edges are traversed in each direction to verify that closed polygons are formed and the polygons are triangulated to form a triangular mesh.

As polygon edge $\mathrm{E}$ is traversed from point $\mathrm{P}$ to point $\mathrm{Q}$, the next edge of the polygon is the next edge counterclockwise in order around point Q. However, if the normals for the neighborhoods are not consistent, the definition of clockwise varies between the neighborhoods. Therefore, the dot product of the normal vectors is used to determine if the next edge should be chosen in a clockwise direction. If so, this direction is maintained in the traversal of the given polygon until another such transition occurs.

\section{RESULTS}

While further investigation is needed to better understand the role of various parameters of the algorithm and locally adaptive approaches should be investigated for neighborhood determination, encouraging results have been obtained with the technique.

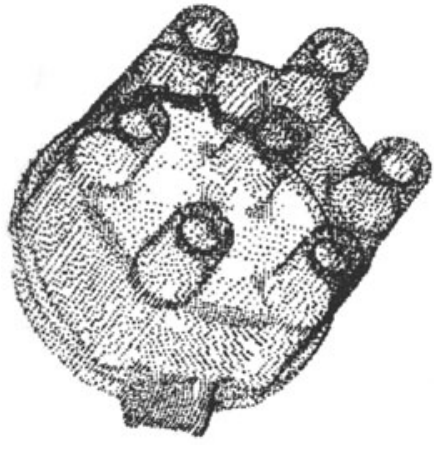

Figure 4. Distributor cap data set

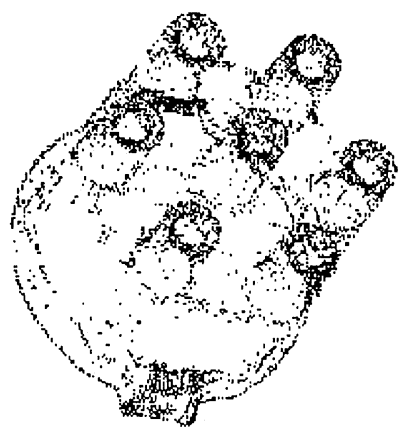

Figure 6. Variation and boundary feature points

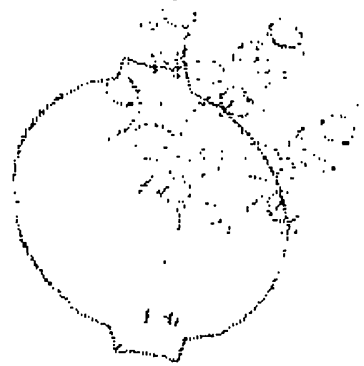

Figure 5. Boundary feature points

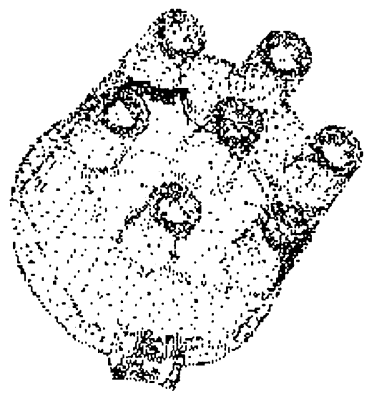

Figure 7. Complementary, variation, and boundary feature points 
The following figures illustrate the results of the algorithm on a distributor cap data set found in Hoppe's work ${ }^{1}$. Figure 4 is the data set consisting of 12,745 points. Figures $5-8$ illustrate the feature point extraction process with Figure 5 displaying 594 boundary feature points, Figure 6 displaying 3,675 feature points after the extraction of variation feature points, Figure 7 displaying 4,101 feature points including complementary points, and Figure 8 displaying the final feature point set after edge extension consisting of 4,347 points.

Figure 9 is the final triangulation that contains 12,635 edges.

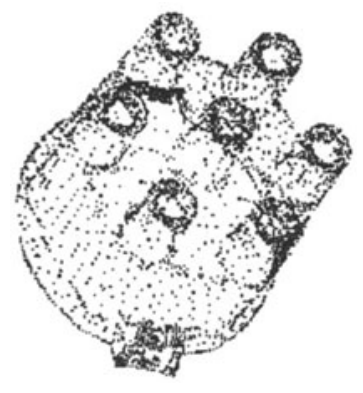

Figure 8. Final feature point set

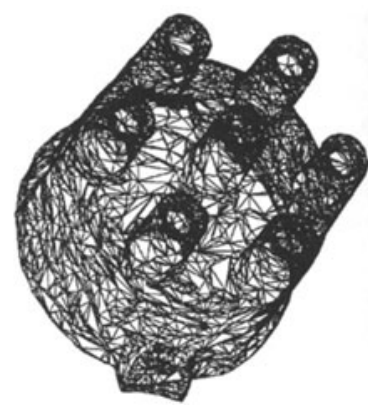

Figure 9. Triangulation of feature points

\section{REFERENCES}

1 Hoppe, Hugues, DeRose, Tony, Duchamp, Tom, McDonald, John and Stuetzle, Werner. Surface Reconstruction from Unorganized Points. Computer Graphics (Proceedings of Siggraph) 26(2), July 1992, 71-78.

2 Hoppe, Hugues, DeRose, Tony, Duchamp, Tom, McDonald, John, and Stuetzle, Werner. Mesh Optimization. Computer Graphics (Proceedings of Siggraph), 1993, 19-26.

3 Kalvin, Alvin D. and Taylor, Russell H.. Superfaces: Polygonal Mesh Simplification with Bounded Error. IEEE Computer Graphics and Applications, May 1996, 64-77.

4 Schroeder, W. J., Zarge, J. A., and Lorensen, W. E.. Decimation of Triangle Meshes. Computer Graphics (Proceedings of Siggraph), 26(2), July 1992, 65-70.

5 Hoppe, Hugues, DeRose, Tony, Dechamp, Tom, Halstead, Mark, Jin, Hubert, McDonald, John, Schweitzer, Jean, and Steutzle, Werner. Piecewise Smooth Surface Reconstruction, Computer Graphics (Proceedings of Siggraph), 1994, 295-302.

6 Bajaj, Chandrajit L., Bernardini, Fausto, and Xu, Guoliang. Automatic Reconstruction of Surfaces and Scalar Fields from 3D Scans. Computer Graphics (Proceedings of Siggraph), 1995, 109-118.

7 Amenta, Nina, Bern, Marshall, and Kamvysselis, Manolis. A New Voronoi-Based Surface Reconstruction Algorithm, Computer Graphics (Proceedings of Siggraph), 1998, 415-421.

8 Garey, Michael R. and Johnson, David S.. Computers and Intractibility, W. H. Freeman and Company, 1979. 